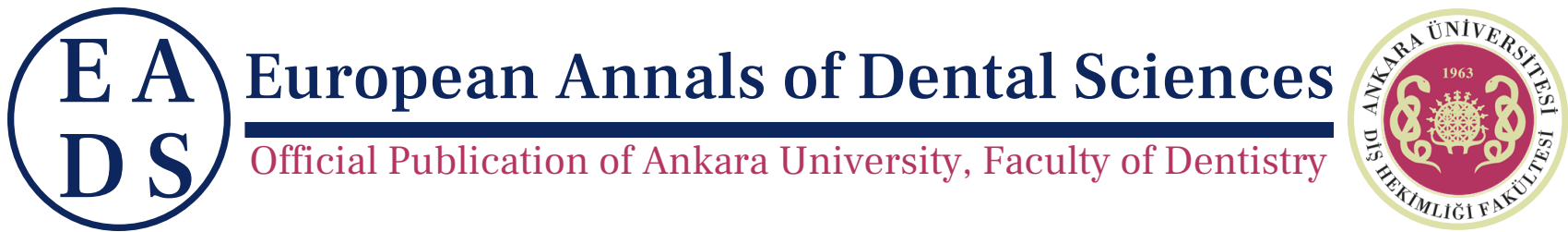

EADS, 2021, $48(3), 129-134$

\title{
Examination Of Damage on Enamel Surface Due to Removal of Metal and Ceramic Brackets: A Review
}

\author{
Hasan Gündal ${ }^{1}$ ** $^{2}$ and Burçak Kaya ${ }^{2}$ \\ ${ }^{1}$ DDS, Başkent University, Faculty of Dentistry, Department of Orthodontics, Ankara, Turkey and ${ }^{2}$ DDs, PhD, \\ Başkent University, Faculty of Dentistry, Department of Orthodontics, Ankara, Turkey \\ *Corresponding Author; hasangundal@gmail.com
}

\begin{abstract}
During fixed orthodontic treatment, there is great possibility that the dental enamel could be damaged due to the process of debonding the bracket. Enamel cracks are one of the most common aspects that can develop or increase during debonding. This review aimed to conduct an examination of cracks in the enamel that occur both prior to and following metal or ceramic brackets being debonded.
\end{abstract}

Key words: ceramic brackets; enamel cracks; debonding; metal brackets

\section{Introduction}

During fixed orthodontic treatment, there is great possibility that the dental enamel could be damaged due to the process of debonding the bracket. Enamel cracks are one of the most common aspects that can develop or increase during debonding. ${ }^{1-3}$ It is known that enamel cracks may result in a reduction in the integrity of the enamel. They can cause stains, plaque accumulation, weakness of the enamel surface, and carious lesions, and they can also take away from the appearance of the teeth. 4,5

The literature comprises a number of studies regarding this concern. Some researchers have investigated different types of bracket bases in terms of retention. ${ }^{6-10}$ Others have evaluated different debonding methods in order to find the one that would result in less damage being done to the enamel, ${ }^{11-14}$ and some have evaluated the bonding strength of metal and ceramic brackets, ${ }^{6,7}$ showing that enamel cracks are associated with the bonding strength. This review aimed to conduct an examination of cracks in the enamel that occur both prior to and following metal or ceramic brackets being debonded.

\section{Brackets}

Orthodontic brackets are small fixed devices that are used for levelling and aligning the teeth. ${ }^{9}$ They are often used to correct malocclusions, such as increased or reversed overjets, crowded teeth, open bites, cross bites, deep bites, and a number of other flaws that occur in the jaw and teeth. Dental brackets are quite commonly used in combination with other orthodontic appliances to aid in the correction of jaw discrepancies while aligning the teeth at the same time.

\section{Evolution of the Brackets}

Orthodontic brackets that have been bonded to the surface of the enamel can provide a tool that can be used in the transfer of the force that is applied by the activated arch wire to the teeth. ${ }^{15} \mathrm{As}$ part of the original approach toward treatment, a slot was used and then attached onto the stainless-steel band. This comprised the gold-standard of practice that was used until late into the1970s. At that time, a new and widely-accepted practice began to be used, which entailed direct bonding of the bracket onto the enamel, which became the standard method that was in lieu of the banding. ${ }^{16}$

The next step in the evolution of brackets included modifying the base design, so as to provide increased bond strength through the use of adhesives. ${ }^{17}$ Aesthetic brackets became very popular as a result of the fact that the number of adults who required orthodontic treatment increased significantly. This treatment population dramatically increased in the middle of the1980s, to the point that adults consisted of $25 \%$ of the total percentage of orthodontic patients when a comparison was made with the much lower number of adult patients that existed during the 1970 s. $^{18}$

\section{Metal Brackets}

Stainless-steel brackets have been in use for a number of decades now and have been shown to have clinically successful results. ${ }^{19}$ 
The bracket base morphology comprises mesh made of metal and can provide sufficient adhesive bond strength to the surface of the enamel surface, so as to meet the demand of the forces that result orthodontically. Research studies that were conducted early on, including that conducted by Gwinnett, ${ }^{20}$ presented reports that the size of the mesh was optimum for attaining increased bond strength. A number of extensive analyses were conducted regarding the rheological properties of the adhesive pastes that were used, in which it was reported that the viscosity is, to a great extent, controlled by the volume of the filler.

\section{Ceramic Brackets}

Even though the term ceramics comprises the inclusion of different compounds, currently, most of the ceramic brackets that are available today comprise aluminum oxide. Basically, there are 2 bracket types, and the production of these brackets entails 2 manufacturing processes that are very different from each other. ${ }^{21}$

The process that is used to make polycrystalline brackets entails the use of fused or sintered particles of aluminum oxide. During this process, the particles are first are blended in a blender. Next, the mixture is molded in such a way that the critical bracket parts can be rammed. The part that was molded is then subjected to a firing process, at a temperature that facilitates burning of the binder and fusing, but not melting, of the aluminum oxide particles. This process of firing is known as sintering.

Aluminum oxide is also used in the manufacturing of monocrystalline ceramic brackets. This process first entails a melting process involving the oxide particles, after which they are slowly cooled down ${ }^{19}$ Monocrystalline bracket is shaped into a similar form as that of a single aluminum oxide crystal. This manufacturing process is much more expensive and difficult due to the fact that this ceramic material is so hard. ${ }^{20}$ The milling process, as well as the presence of sharp corners, results in stresses being placed on the material, and it also predisposes the brackets to the formation of fractures.

\section{Types of Retention Mechanisms Incorporated in the Ce- ramic Bracket Base}

Aluminum oxide is used to manufacture ceramic brackets, and it is a material that is inert. As a result of this, it is not able to chemically form direct adhesion to any of the resins that are used for bonding that are currently available. As a result, there are two basic mechanisms that have been developed in which brackets made of ceramic can be attached to adhesives. ${ }^{22}$

The first of these methods is mechanical retention, which is achieved as a result of recesses or indentations that exist in the base of the bracket, in very much the same way as the mesh that is on the metal bracket base. The benefit of these indentations is that they can provide a kind of mechanical interlocking between the bracket and the adhesive resin. The second method entails the use of an intermediate glass layer at the bracket base, which is done using a silane coupler. This allows for the facilitation of a chemical bond to form between the bracket and the resin that is used as the adhesive. Hence, there are 3 very different mechanisms that can be used for retention when attaching brackets that are made of ceramic to the agent used for bonding. These are 1) chemical retention, which is performed using silane, 2) mechanical retention, and 3) the combination of both methods. ${ }^{23}$

\section{Effects of the Retention Mechanism on Bond Strengths}

The possibility of enamel damage occurring during the process of the debonding of ceramic brackets may be the result of numerous factors. One of these significant factors comprises an increase in the bond strength at the interface of the bracket and the adhesive. A number of the studies that have been conducted have reported the fact that ceramic brackets that have been retained chemically provide a bonding strength that is significantly stronger when compared with conventional metal brackets. ${ }^{24,25}$ This increased bonding strength, as a result of the use of ceramic brackets, thus results in the bond failing to form at the enamel-to-adhesive interface, instead of at the 'safer' bracket-to-adhesive interface, which occurs quite commonly with brackets that are made of metal. When a bond fails to form at the enamel-to-adhesive interface, this causes a higher number of enamel fractures to occur, $3,26,27$ which is of great concern for the patients receiving the procedure, the clinicians performing the procedure, and the manufacturers who are producing the appliances.

\section{Advantages and Disadvantages of Ceramic Brackets}

There is only one advantage to ceramic brackets, which is their aesthetics. ${ }^{28}$ On the other hand, there are many disadvantages to ceramic brackets, which include the following:

1) There is a greater chance that fracturing will occur during the process of debonding the brackets, especially with the traditional techniques that are used for debonding.

2) Brackets such as these are not able to endure the extensive torsional forces that are in play, especially after the bracket surface has been notching during treatment.

3) Their use should be avoided on teeth that are vulnerable. Hence, it is necessary for clinicians to conduct a very thorough pre-treatment examination, specifically with regard to the enamel surface characteristics, via transillumination. This will allow for the detection of any cracks or fractures, or any other defects with the ability to become the sites of possible enamel fracture during the process of debonding brackets. An examination such as this should be considered as risk management prior to the orthodontic treatment.

4) If the brackets come into contact with opposing tooth surfaces, this will result in enamel wear. Hence, ceramic bracket placement should not be performed on the lower anterior teeth under circumstances in which the patient has a deep overbite with minimal overjet. In cases such this, it is necessary to create sufficient overjet prior to bonding of the lower incisors. In the same way, during the process of retraction of the maxillary incisors, it is necessary to first reduce the overbite, which will prevent the maxillary incisors from coming into contact with the ceramic mandibular brackets.

5) It is also possible for these brackets to result in notches being created in the arch wires. This would result in greater friction occurring between the arch wire and the bracket. This, in turn, may result in decreased tooth movement efficiency.

6) Using these brackets should be avoided for patients who will undergo orthognathic surgery. Fractures in these brackets either prior to, during, or following the surgery, may potentially result in the occurrence of undesirable complications.

7) As a result of the potential fracture of enamel of the bracket, clinicians should be extra careful in the debonding of ceramic brackets.

\section{Bonding Procedure}

In dentistry, the application of adhesive bonding resins has increased significantly as a result of the introduction of a technique that is known as enamel acid-etching, which was introduced by Buonocore in $1955 .{ }^{29}$ Through the demonstration of a 100X increase in retention onto teeth that had been etched for $30 \mathrm{~s}$ via the use of $85 \%$ phosphoric acid, Buonocore was able to develop modern dentistry techniques with regard to adhesives. ${ }^{29}$ Over the last fifty 
years, bonding using a number of adhesive resins to enamel has been researched in orthodontic studies. The technique of direct bonding was centered around the notion of the attachment of orthodontic appliances onto the enamel surface through the use of adhesives. Since then, this technique has become the foundation that contemporary orthodontics has been built on.

Even though the techniques and materials that are used for the bonding of the bracket have changed throughout the years, the procedure has mainly remained the same. In general, orthodontic bonding comprises a three-step technique, which consists of an etchant, primer, and adhesive. However, more recently, these three steps have often been combined, thus resulting in only two steps, or even just a single step. Research has revealed that the microporosities resulting from the acid-etching process make possible the incorporation of small resin tags into the surface of the enamel. This occurrence results in microscopic mechanical interlocks being created between the resin and the enamel. ${ }^{26,30,31}$ The concept of adhesion has been extensively studied, and at the current time, there are a combination of theories, such as mechanical, adsorption, diffusion, and electrostatic, that are used in the explanation of this phenomenon. ${ }^{32}$ The conventional process of acid-etch bonding comprises 4 steps, as follows: 1 ) cleansing of the enamel surface; 2) conditioning of the enamel, i.e. etching;3) the application of a primer, and 4) the application of the adhesive. The purpose behind cleansing of the enamel surface is to remove any contaminants or debris that could possibly prevent the conditioner and/or primer materials from coming into contact with the enamel surface while applying them. Most often, cleansing of the enamel surface is accomplished by applying a rubber polishing bur and pumice for $10 \mathrm{~s}$ per tooth. Conditioning of the enamel, i.e. etching, has generally been accomplished via the use of $37 \%$ orthophosphoric acid, which is capable of dissolving enamel prisms, which is necessary to create the microporosities that are required for development of the resin tags. The application of a primer is necessary to aid in diffusing the adhesive monomers into entire depth of the etching on the enamel surface. Following this, an adhesive resin is applied, which can be either light-curing or self-curing. Following this standardized procedure allows clinicians to attain successful direct or indirect bonding of the orthodontic brackets and other appliances onto the teeth, with bonding strength that is sufficient to withstand the majority of the forces that commonly occur during orthodontic treatment within the oral cavity. 33

\section{Debonding Procedure}

The methods used to remove the bonded orthodontic brackets involve either deforming the bracket wings or the bracket base, and 'peeling' the loosened bracket from the tooth, or applying a shear force across the adhesive. Detachment of metal orthodontic brackets with ligature cutters is considered by some clinicians to result in the least amount of enamel damage. ${ }^{34}$ Adhesive failure can occur in the resin used as the adhesive, which is known as cohesive failure, at any interface between the bracket/adhesive/enamel or at a number of different sites. ${ }^{35}$ Factors that may influence the site of detachment are the procedure used to prepare the tooth for bonding, the type of bonding material, and the type of instrument used to remove the brackets. ${ }^{36}$

Heravi et al. ${ }^{1}$, reported the use of three different pliers for the process of debonding metal brackets, which comprised a bracket remover with a single blade, a bracket remover with a double blade, and an orthodontic ligature cutter. The results of their study showed that all of these methods caused adhesive failure, either at the interface of the bracket base-to-adhesive or within the adhesive resin (cohesively). Additionally, the number of enamel cracks increased. Therefore, they concluded that despite the widespread use of these methods for debonding, it is necessary to define methods that will result in there being less damage to the enamel.

\section{Debonding methods for ceramic brackets}

As a result of the brittle nature of brackets made of ceramic, the earlier methods that were used for mechanical debonding very often resulted in failure of the enamel or bracket. As a result of this occurrence, researchers, manufacturers, and clinicians have aimed at developing new and improved techniques for debonding that are specially designed for use with brackets made of ceramic. These new techniques for debonding have included ultrasonic, mechanical, laser, and electrothermal.

\section{Mechanical debonding}

The earliest debonding instruments used on brackets made of ceramic comprised the application of heavy shear torsion forces to teeth that were already mobile and sensitive. The nature of the sudden fractures in the brackets that was associated with these methods resulted in the potential occurrence of fracturing or cracks in the enamel. ${ }^{19}$ It has been stated that "This method of force concentration is analogous to the delamination of two pieces of bonded wood. Attempting to twist one piece from the other will require great forces. Wedging a chisel at the interface of the two will usually be less destructive and require significantly less force to separate."

At the present time, among the most popular techniques for the mechanical debonding of brackets made of ceramic is one that entails the application of debonding plier blades near the surface of the enamel, but still within the adhesive. ${ }^{15}$ In a normal in-vitro shearbond strength test, however, the force must be applied to one side of the bracket. ${ }^{25}$ Hence, it has been necessary to attempt to simulate this situation in a clinical setting so as to measure the actual force that is being applied by the pliers throughout the debonding procedure, via the application of this force on both sides of the bracket at the bracket-to-adhesive interface. ${ }^{32}$

The application of the load, simultaneously, to both sides using pliers causes an increased risk that a crack will be created in the brittle adhesive. Recently conducted studies have reported that performing this method results in the transmission of one-third less force onto the enamel when a comparison was made with a pure shear force. This is a very significant reduction in the debonding force, which, in turn, puts much less stress on the surface of the enamel, which reduces the chance that fracture damage will occur.

The plier blade width can have an effect on the debonding forces. Either narrow, 2.0-mm, blades or the wide, 3.2- $\mathrm{mm}$, blades can be used by the clinician when debonding a bracket made of ceramic with a debonding instrument that has a sharp edge. Using narrow blades means that there is lower debonding stress, at about 120 $\mathrm{kg} / \mathrm{cm} 2$, than what would occur when using wider blades, which would result in $150 \mathrm{~kg} / \mathrm{cm} 2$. That is to say, a reduction in the debonding force occurs when narrow plier blades are used.

\section{Ultrasonic debonding}

The ultrasonic technique uses specially designed tips applied at the bracket-adhesive interface to erode the adhesive layer between the enamel surface and bracket base. ${ }^{36}$ The force magnitudes required with the ultrasonic approach are significantly lower than those required for the conventional methods of bracket removal. However, the ultrasonic technique has a major disadvantage. The debonding time using this technique is 30 to $60 \mathrm{~s}$ per bracket, which is significantly more when compared with 1 to $5 \mathrm{~s}$ for other bracket removal methods. In addition, there is excessive wear of the relatively expensive ultrasonic tips. This wear is the result of the friction between the softer steel tip moving against the much harder ceramic surface. ${ }^{23}$ There is also the potential for gouging the enamel surface during the erosion process. Consequently, this method of removal for brackets made of ceramic has recently not been recommended for clinical use. 


\section{Laser debonding (electrothermal debonding)}

The application of laser technology in dentistry was not considered practical until recently, because early research had shown that laser irradiation of teeth generated too much heat, which resulted in pulpal damage and enamel fracturing. ${ }^{37}$ Within the past few years, significant advances in laser technology and in our understanding of how the energy in a laser light beam interacts with certain materials has greatly decreased the undesirable thermal effects. Hence, these developments have resulted in the successful application of lasers in dentistry. ${ }^{32}$ This new knowledge has allowed the selection of a laser with the proper characteristics to assure the rapid debonding of brackets made of ceramic without damaging the tooth, the bracket, or the soft tissues. With the development of such systems, bonding strengths can be maximized, facilitating both the use of new treatment modalities and small brackets with larger forces, without the fear of undesirable damage during debonding. ${ }^{38,39}$

Any process that degrades the bonding resin will facilitate debonding. Laser-initiated degradation can occur as the result of thermal softening, thermal ablation, and photoablation. Thermal softening, which occurs at relatively low rates of laser energy deposition, heats the bonding agent up until it softens, and the brackets debond by sliding off the tooth. Heating could occur directly in the resin, the bracket, or the tooth, depending on how these components absorbed the light energy. Since the process is relatively slow, it would be expected that this type of debonding would result in a significant increase in both the tooth and the bracket temperature.

Debonding brackets made of ceramic has been attempted using both $\mathrm{CO} 2$ and YAG lasers in combination with mechanical torque. The use of a laser is conceptually similar to the use of the electrothermal approach, that is, through heat generation to soften the adhesive. With the laser, the torque force required to debond polycrystalline brackets was lowered by a factor of 27 for molars and a factor of 16 for incisors when compared with the mechanical debonding forces used without the laser. The polycrystalline brackets were illuminated for $2 \mathrm{~s}$ with a focused $\mathrm{CO} 2$ laser beam of $14 \mathrm{~W}$, whereas the monocrystalline brackets needed only half that amount of energy. The laser approach, although still experimental, is more precise with regard to time and the amount of heat application, and therefore would have better control of the amount of heat transmitted to the tooth. A major disadvantage, in addition to the side effects of the thermal energy on the pulp, is the high cost of the laser instrument.

The short- and long-term effects of laser (electrothermal) debonding on the underlying pulp and the degree of patient discomfort have been examined in some studies. ${ }^{40-44}$ Forty-eight premolars planned for orthodontic extraction were bonded with monocrystalline brackets and debonded using the electrothermal (laser) instrument. Patients were questioned about the sensations during debonding. The teeth were extracted at 1 or 4 weeks after bracket removal and were then histologically examined. The findings indicated that at the end of 1 week, the predominant inflammatory cells were lymphocytes, with no pulpal necrosis observed. At 4 weeks, chronic inflammation decreased over time, indicating repair of the damaged areas. The odontoblast tic layer was intact, although some evidence of the earlier damage remained in $12 \%$ of the specimens. A similar reaction occurs when a tooth has a cavity preparation. The histological evidence indicated that the pulp damage was mostly reversible, and the pulp injury, when it occurred, was relatively mild in the premolar teeth. In the clinical tests, the patients experienced minimum discomfort. Generally, the sensation was described as "warmer than normal body temperature," but was well-tolerated. 45

\section{Enamel cracks}

The origin of the cracks that have been observed in the enamel of human teeth has been a subject of great controversy, as these cracks are believed to be multi-causal. According to the current literature, there are two basic possibilities for this, which comprise: 1) developmental disturbances and 2) some type of mechanical failure. ${ }^{39}$ Developmental disturbances imply that there was a fault in the development of one of the ameloblast sectors, while mechanical failure implies that there was a fracture that occurred in the enamel cap at some point, either prior to or following the eruption. High stress placed on the surface of the tooth, as a result of mechanical loads or fast thermal changes, are transferred onto the dentinoenamel junction. Enamel cracks may occur as a result of the evident difference that exists between the rigidity in the enamel and the dentin. ${ }^{34}$ In addition, traumatic injury that occurs directly to the teeth may also cause cracks. Fractures such as this generally occur in the direction of the enamel prisms, and usually do not surpass the enamel-dentin border. They usually do not require treatment, with the exception of a vitality check-up at 6 to 8 weeks following the procedure.

\section{The incidence of enamel cracks after mechanical debond- ing}

Zachrisson et al. ${ }^{46}$ analyzed and compared the prevalence and appearance of enamel cracks in teeth of 2 post-orthodontic groups and a matched untreated group of adolescents. Using fiber-optic transillumination, they made a clinical assessment of enamel cracks (prevalence, localization, expression, direction) in the three groups of adolescents representing debonded, debanded, and orthodontically untreated teeth. The findings indicated that enamel cracks were extremely common in all three groups. Most of the cracks were not prominent and could easily be overlooked on routine clinical examination. The majority of the cracks were vertical and, with the exception of the mandibular incisors, were most frequently localized in the gingival two-thirds of the facial tooth surfaces. The marked cracks were observe mostly on maxillary canines and central incisors in all of the groups. The few horizontal cracks that were noted were mostly located in the maxillary and mandibular central incisors.

Heravi et al. ${ }^{1}$ compared the adhesive remnant scores (ARI) in addition to the number, lengths, and directions of enamel cracks before bonding and after debonding of metal orthodontic brackets with 3 different methods, which included a side cutter, a singleblade bracket remover, or a double-blade bracket remover. After debonding, the number of enamel cracks and pronounced cracks, as well as the lengths of the enamel cracks increased in all of the groups. The ARI scores and the direction of propagation of the enamel cracks were not influenced by the debonding method used. They concluded that the enamel damage caused by the 3 methods proved that the debonding procedures must be realized with caution, and despite the widespread use of these methods, new techniques that result in less enamel damage should be developed.

\section{Incidence of enamel cracks after laser debonding}

Electrothermal (laser) debracketing, requires the application of the hot tip of a soldering gun-like device to the bracket until debonding occurs. Dovgan et al. ${ }^{40}$ used the electrothermal technique for debonding and found that brackets made of ceramic could be removed in less than $4^{\circ} \mathrm{C}$ with no significant pulpal damage according to histologic examination. However, Rueggeberg et al. ${ }^{43}$ found that the time required for the same technique exceeded $1 \mathrm{~min}$, and the bonding interface temperature exceeded $160^{\circ} \mathrm{C}$ for all 9 of the bonding agents tested. They noted that the increased debonding times 
and high interface temperatures, which are characteristics of electrothermal debonding, would be clinically inconvenient and would have the potential to damage the pulp.

Strobl et al. 44 investigated the in vitro removal of both polycrystalline and monocrystalline brackets made of ceramic that were bonded to human premolars and molars with a highly filled composite resin. The debonding torque was applied after the brackets made of ceramic were lased with $\mathrm{CO} 2$ and Nd: YAG lasers. The lasing time was set at $2 \mathrm{~s}$. The results of their study showed that laseraided debonding significantly reduced the debonding force and the risk of enamel damage.

Rickabaugh et al. ${ }^{42}$ investigated the effects of varying lasing times, debonding forces and their effects on intrapulpal temperature increase. The results of their study showed that the tensile debonding force could be reduced by a factor of 12 if an intrapulpal temperature increase of approximately $5.5^{\circ} \mathrm{C}$ was accepted.

Obata ${ }^{41}$ studied the effects of the $\mathrm{CO} 2$ laser on the removal of brackets made of ceramic using 2 different bonding adhesives. After measuring the shear forces, the enamel and bracket fractures were observed in the specimens. The laser-irradiated group, which included the use of either 4-META MMA resin or Bis-GMA resin, had no fractures.

\section{Conclusion}

All clinically and commercially acceptable braces must demonstrate an optimum bonding strength for successful orthodontic treatment. However, ceramic brackets show higher bonding strength when compared to metal brackets. This increases the risk of possible enamel damage during deboning, especially when higher shear bond strength values are achieved. There is a high risk of new enamel crack formation when ceramic brackets are debonded using traditional debonding methods. On the other hand, the risk of new enamel crack formation significantly reduces if debonding of ceramic brackets is realized by using Er: YAG laser. Nevertheless, the side effects of laser irradiation still must be clarified. Further research is required to overcome the problems related with the debonding of orthodontic brackets and enhance the adhesive systems used for the bonding of ceramic brackets especially.

\section{Author Contributions}

H.G; literature review, data collection, interpretation of the findings, writing the manuscript. B.K; planning the methodology, supervising the project, writing the manuscript, reviewing the article.

\section{Conflict of Interest}

The authors of the current article certify that they have no affiliations with any organization or entity with any financial interest.

\section{Authors' ORCID(s)}

H.G. $\quad 0000-0001-8539-9759$

B.K. $\quad 0000-0002-7953-3445$

\section{References}

1. Heravi F, Rashed R, Raziee L. The effects of bracket removal on enamel. Aust Orthod J. 2008;24(2):110-5.

2. Øgaard B, Fjeld M. The enamel surface and bonding in orthodontics. In: Seminars in orthodontics. vol. 16. Elsevier; 2010. p. 37-48.
3. Thompson RE, Way DC. Enamel loss due to prophylaxis and multiple bonding/debonding of orthodontic attachments. Am J Orthod. 1981;79(3):282-95. doi:10.1016/0002-9416(81)900762.

4. Zachrisson BU, Arthun J. Enamel surface appearance after various debonding techniques. Am J Orthod. 1979;75(2):121-7. doi:10.1016/0002-9416(79)90181-7.

5. Zachrisson BU, Skogan O, Höymyhr S. Enamel cracks in debonded, debanded, and orthodontically untreated teeth. Am J Orthod. 1980;77(3):307-19. doi:10.1016/0002-9416(80)900846.

6. Bishara SE, Ostby AW, Laffoon J, Warren JJ. Enamel cracks and ceramic bracket failure during debonding in vitro. Angle Orthod. 2008;78(6):1078-83. doi:10.2319/112007-540.1.

7. Britton JC, McInnes $\mathrm{P}$, Weinberg R, Ledoux WR, Retief DH. Shear bond strength of ceramic orthodontic brackets to enamel. Am J Orthod Dentofacial Orthop. 1990;98(4):348-53. doi:10.1016/s0889-5406(05)81492-1.

8. Guess MB, Watanabe LG, Beck FM, Crall MG. The effect of Silane coupling agents on the bond strength of a polycrystalline ceramic bracket. J Clin Orthod. 1988;22(12):788-92.

9. Hajrassie MK, Khier SE. In-vivo and in-vitro comparison of bond strengths of orthodontic brackets bonded to enamel and debonded at various times. Am J Orthod Dentofacial Orthop. 2007;131(3):384-90. doi:10.1016/j.ajodo.2005.06.025.

10. Viazis AD, Cavanaugh G, Bevis RR. Bond strength of ceramic brackets under shear stress: an in vitro report. Am J Orthod Dentofacial Orthop. 1990;98(3):214-21. doi:10.1016/s08895406(05)81598-7.

11. Bishara SE, Fehr DE. Comparisons of the effectiveness of pliers with narrow and wide blades in debonding ceramic brackets. Am J Orthod Dentofacial Orthop. 1993;103(3):2537. doi:10.1016/0889-5406(93)70006-a.

12. Bishara SE, Fonseca JM, Boyer DB. The use of debonding pliers in the removal of ceramic brackets: force levels and enamel cracks. Am J Orthod Dentofacial Orthop. 1995;108(3):242-8. doi:10.1016/s0889-5406(95)70016-1.

13. Boyer DB, Engelhardt G, Bishara SE. Debonding orthodontic ceramic brackets by ultrasonic instrumentation. Am J Orthod Dentofacial Orthop. 1995;108(3):262-6. doi:10.1016/s08895406(95)70019-6.

14. Feldon PJ, Murray PE, Burch JG, Meister M, Freedman MA. Diode laser debonding of ceramic brackets. Am J Orthod Dentofacial Orthop. 2010;138(4):458-462. doi:10.1016/j.ajodo.2008.11.028.

15. Oliver RG. The effect of different methods of bracket removal on the amount of residual adhesive. Am J Orthod Dentofacial Orthop. 1988;93(3):196-200. doi:10.1016/s0889-5406(88)800039.

16. Harris AM, Joseph VP, Rossouw PE. Shear peel bond strengths of esthetic orthodontic brackets. Am J Orthod Dentofacial Orthop. 1992;102(3):215-9. doi:10.1016/s0889-5406(05)81055-8.

17. Harris AM, Joseph VP, Rossouw E. Comparison of shear bond strengths of orthodontic resins to ceramic and metal brackets. J Clin Orthod. 1990;24(12):725-8.

18. Mewman G. Bonding plastic orthodontic attachments to tooth enamel. J NJ Dent Soc. 1964;35:346-358.

19. Fernandez L, Canut JA. In vitro comparison of the retention capacity of new aesthetic brackets. Eur J Orthod. 1999;21(1):717. doi:10.1093/ejo/21.1.71.

20. Gwinnett AJ. A comparison of shear bond strengths of metal and ceramic brackets. Am J Orthod Dentofacial Orthop. 1988;93(4):346-8. doi:10.1016/0889-5406(88)90165-5.

21. Swartz ML. Ceramic brackets. J Clin Orthod. 1988;22(2):82-8.

22. Wang WN, Meng CL, Tarng TH. Bond strength: a comparison between chemical coated and mechanical interlock bases of ceramic and metal brackets. Am J Orthod Dentofacial Orthop. 1997;111(4):374-81. doi:10.1016/s0889-5406(97)80019-4. 
23. Bishara SE, Fehr DE. Ceramic brackets: something old, something new, a review. Semin Orthod. 1997;3(3):178-88. doi:10.1016/s1073-8746(97)80068-0.

24. Bishara SE, Olsen ME, Von Wald L. Evaluation of debonding characteristics of a new collapsible ceramic bracket. Am J Orthod Dentofacial Orthop.1997;112(5):552-9. doi:10.1016/s08895406(97)70083-0.

25. Díaz C, Swartz M. Debonding a new ceramic bracket: a clinical study. J Clin Orthod. 2004;38(8):442-5.

26. Elekdag-Turk S, Isci D, Ozkalayci N, Turk T. Debonding characteristics of a polymer mesh base ceramic bracket bonded with two different conditioning methods. Eur J Orthod. 2009;31(1):84-9. doi:10.1093/ejo/cjno67.

27. Joseph VP, Rossouw E. The shear bond strengths of stainless steel and ceramic brackets used with chemically and lightactivated composite resins. Am J Orthod Dentofacial Orthop. 1990;97(2):121-5. doi:10.1016/0889-5406(90)70084-p.

28. Esthetic Moieties. Journal of Esthetic and Restorative Dentistry. 1993;5(1):37-37. doi:https://doi.org/10.1111/j.17088240.1993.tboo742.x.

29. Buonocore $M$ G. A simple method of increasing the adhesion of acrylic filling materials to enamel surfaces. J Dent Res. 1955;34(6):849-53. doi:10.1177/00220345550340060801.

30. Gwinnett AJ, Buonocore MG. ADHESIVES AND CARIES PREVENTION; A PRELIMINARY REPORT. Br Dent J. 1965;119:77-80.

31. Gwinnett AJ, Matsui A. A study of enamel adhesives. The physical relationship between enamel and adhesive. Arch Oral Biol. 1967;12(12):1615-20. doi:10.1016/0003-9969(67)90195-1.

32. Summitt JB, Robbins JW, Schwartz RS. Fundamentals of operative dentistry: a contemporary approach. Quintessence Publ. Comp. Inc.; 2001.

33. Eliades $\mathrm{T}$, Viazis $\mathrm{AD}$, Eliades $\mathrm{G}$. Bonding of ceramic brackets to enamel: morphologic and structural considerations. Am J Orthod Dentofacial Orthop. 1991;99(4):369-75. doi:10.1016/08895406(91)70020-w.

34. Coups-Smith KS, Rossouw PE, Titley KC. Glass ionomer cements as luting agents for orthodontic brackets. Angle Orthod. 2003;73(4):436-44. doi:10.1043/00033219(2003)073<0436:Gicala>2.0.Co;2.

35. Joseph VP, Rossouw PE. The shear bond strengths of stainless steel orthodontic brackets bonded to teeth with orthodon- tic composite resin and various fissure sealants. Am J Orthod Dentofacial Orthop. 1990;98(1):66-71. doi:10.1016/08895406(90)70033-9.

36. Englehardt G, Boyer D, Bishara S. Debonding orthodontic ceramic brackets by ultrasonic instrumentation. Journal of Dental Research. 1993;72(3):139-145.

37. Keller U, Hibst R. Experimental studies of the application of the Er:YAG laser on dental hard substances: II. Light microscopic and SEM investigations. Lasers Surg Med. 1989;9(4):345-51. doi:10.1002/lsm.1900090406.

38. Chirila TV, Constable IJ, van Saarloos PP, Barrett GD. Laserinduced damage to transparent polymers: chemical effect of short-pulsed (Q-switched) Nd:YAG laser radiation on ophthalmic acrylic biomaterials. I. A review. Biomaterials. 1990;11(5):305-12. doi:10.1016/0142-9612(90)90106-z.

39. Yow L, Nelson JS, Berns MW. Ablation of bone and polymethylmethacrylate by an $\mathrm{XeCl}$ (308 nm) excimer laser. Lasers Surg Med. 1989;9(2):141-7. doi:10.1002/1sm.1900090209.

40. Dovgan JS, Walton RE, Bishara SE. Electrothermal debracketing: patient acceptance and effects on the dental pulp. Am J Orthod Dentofacial Orthop. 1995;108(3):249-55 doi:10.1016/s0889-5406(95)70017-x.

41. Obata A. Effectiveness of $\mathrm{CO} 2$ laser irradiation on ceramic bracket debonding. J Jpn Orthod Soc. 1995;54:285-295.

42. Rickabaugh JL, Marangoni RD, McCaffrey KK. Ceramic bracket debonding with the carbon dioxide laser. Am J Orthod Dentofacial Orthop. 1996;110(4):388-93. doi:10.1016/s08895406(96)70040-9.

43. Rueggeberg FA, Lockwood P. Thermal debracketing of orthodontic resins. Am J Orthod Dentofacial Orthop. 1990;98(1):56-65. doi:10.1016/0889-5406(90)70032-8.

44. Strobl K, Bahns TL, Willham L, Bishara SE, Stwalley WC. Laseraided debonding of orthodontic ceramic brackets. Am J Orthod Dentofacial Orthop. 1992;101(2):152-8. doi:10.1016/08895406(92)70007-w.

45. Coley-Smith A, Rock WP. Distortion of metallic orthodontic brackets after clinical use and debond by two methods. Br J Orthod. 1999;26(2):135-9. doi:10.1093/ortho/26.2.135.

46. Zarrinnia K, Eid NM, Kehoe MJ. The effect of different debonding techniques on the enamel surface: an in vitro qualitative study. Am J Orthod Dentofacial Orthop. 1995;108(3):284-93. doi:10.1016/s0889-5406(95)70023-4. 